\title{
¿Conocemos todas las interacciones farmacológicas? El transportador OATP1B1
}

\author{
Lara María Martínez Celdran', Javier Guevara Ferrando', Lucrecia Moreno Royo² \\ 1. Alumno $5^{\circ}$ curso farmacia en la Universidad Cardenal Herrera - CEU. Alfara del Patriarca. Valencia. 2. Catedrática de farmacología y Vicedecana en \\ la Facultad de Ciencias de la Salud. Universidad Cardenal Herrera - CEU. Alfara del Patriarca. Valencia.
}

\section{PALABRAS CLAVE}

Interacciones 0ATP1B1, transporte activo, inhibición competitiva,

Olmesartán, Rosuvastatina, miositis

\section{KEYWORDS}

OATP1B1 Interactions, active transport, competitive inhibition, Olmesartan, Rosuvastatin, myositis

\section{RESUMEN}

En ancianos es frecuente la polimedicación. Esto incrementa el riesgo de sufrir reacciones adversas a los medicamentos, y también el riesgo de sufrir interacciones que pueden ser relevantes.

Las interacciones más frecuentes son las que afectan a la farmacocinética de los medicamentos y, especialmente, al metabolismo de estos. Aquí el citocromo P450 tiene mucha relevancia, pero desde hace poco más de una década se conoce otro mecanismo implicado, las proteínas transportadoras de membrana. Dentro de estas tienen especial relevancia las OATP (Organic anion transporting polypeptide) de las que existen diferentes tipos y ubicaciones. La competición de diferentes substratos por estas proteínas puede generar interacciones que acaban repercutiendo en el tratamiento farmacoterapeutico del paciente.

Do we know all the pharmacological interactions? The OATP1B1 transporter

\section{ABSTRACT}

In the elderly, polymedication is frequent. This increases the risk of adverse reactions to medications, and also the risk of interactions that may be relevant. The most frequent interactions are those that affect the pharmacokinetics of drugs and, especially, their metabolism. Here the cytochrome P450 has a lot of relevance, but for a little more than a decade we know another mechanism involved, the membrane transporter proteins. Within these are the OATP (Organic anion transporting polypeptide) of which there are different types and locations. The competition of different substrates for these proteins can generate interactions that end up having an impact on the pharmacotherapeutic treatment of the patient.

\section{Introducción}

La incidencia de las enfermedades crónicas guarda una relación directamente proporcional al creciente envejecimiento de la población. Es muy frecuente observar en la población anciana la coexistencia de comorbilidades que derivan a la prescripción de varios medicamentos. También, otros factores demográficos y socio sanitarios (sexo, nivel cultural, situación económica, prescripción inapropiada o intervención de varios médicos, etc.) contribuyen a que el paciente anciano sea más susceptible a la polifarmacia con el consiguiente riesgo de presentar interacciones farmacológicas, además de las reacciones adversas propias de cada medicamento $(1,2)$.

La polimedicación constituye en definitiva un problema de salud pública. Esta situación desencadena una serie de consecuencias negativas, tales como el mayor riesgo de caídas y hospitalización, de hecho, estudios recientes han estimado entre un 10$20 \%$ de los ingresos hospitalarios es debido a una interacción medicamentosa (3).
Recibido: $30-11-2018$

Aceptado: 18-12-2018

Disponible online: 28-12-2018
Financiación: ninguna.

Conflicto de intereses: ninguno.

Cite este artículo como: Martínez LM, Guevara J, Moreno L. ¿Conocemos todas las interacciones farmacológicas? el transportador OATP1B1. Farmacéuticos Comunitarios. 2018 Dec 28; 10(4):29-32. doi:10.5672/FC.2173-9218.(2018/ Vol10).004.06

Correspondencia: Lucrecia Moreno Royo (Imoreno@uch.ceu.es)

ISSN 1885-8619 @ SEFAC (Sociedad Española de Farmacia Familiar y Comunitaria). Todos los derechos reservados. 


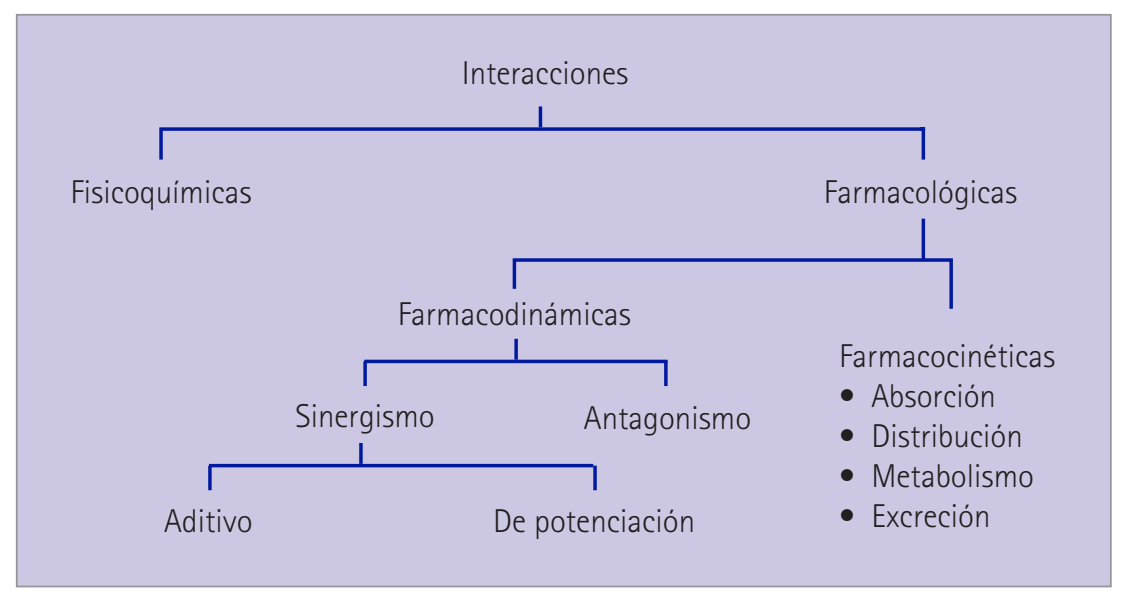

Figura 1 Clasificación de las interacciones con medicamentos

Las interacciones se definen como alteraciones de la actividad o efecto de un fármaco, tanto a nivel cualitativo como cuantitativo, y pueden surgir como consecuencia de una interacción fármaco-alimento, fármaco-fármaco y/o fármaco-complemento dietético o planta medicinal.

Como se observa en la figura 1, las interacciones las podemos clasificar atendiendo a su naturaleza en:

1. Interacciones fisicoquímicas: aquellas que atañen a los aspectos galénicos durante la fabricación del medicamento (la solubilización de la forma farmacéutica...) y que, posteriormente, afecta a la liberación del principio activo.

2. Interacciones farmacológicas que, a su vez se subdividen en:

2.1. Interacciones farmacodinámicas, aquellas que tienen lugar en la biofase. Un fármaco interfiere en la actividad del otro al actuar en el mismo lugar de acción (receptor, enzima, etc.), siendo las posibles respuestas (tabla 1):

2.1.1. Sinergismo de suma: aumenta la actividad farmacológica debido a la suma de los efectos individuales de cada fármaco.
2.1.2. Sinergismo de potenciación: igualmente aumenta la actividad farmacológica, en este caso el efecto es superior a la suma de los efectos de cada fármaco implicado.

2.1.3. Antagonismo: se produce una reducción o anulación del efecto del fármaco, debido a la interacción.

2.2. Interacciones farmacocinéticas. Estas interacciones se producen porque un fármaco modifica los procesos a nivel de la absorción, distribución, metabolismo o excreción de otro fármaco.

En la tabla 2 se ilustra la etapa farmacocinética implicada del proceso ADME así como las modificaciones que pueden llevar a cabo algunos fármacos en cada una de las etapas:

En cuanto a las interacciones con alimentos, un ejemplo clásico es la administración conjunta de IMAO (inhibidores de la mono-aminoxidasa mitocondrial) con alimentos ricos en tiramina, como el queso, que desencadena una crisis hipertensiva, una reacción adversa de relevancia clínica.

También puede disminuir el efecto del fármaco, como es el caso de la

Tabla 1 Tipos de interacciones farmacodinámicas

\begin{tabular}{|l|l|l|}
\hline \multicolumn{1}{|c|}{ Interacciones farmacodinámicas } & \multicolumn{1}{|c|}{ Ejemplos } & \multicolumn{1}{|c|}{ Efecto } \\
\hline Sinergismo de suma & $\begin{array}{l}\text { Gentamicinal } \\
\text { tobramicina y } \\
\text { cefalotina }\end{array}$ & $\begin{array}{l}\text { Aumento efectos } \\
\text { adversos (aumento } \\
\text { nefrotoxicidad). }\end{array}$ \\
\hline Sinergismo de potenciación & $\begin{array}{l}\text { Trimetroprim y } \\
\text { sulfametoxazol }\end{array}$ & $\begin{array}{l}\text { La actividad } \\
\text { antimicrobiana es } \\
\text { superior a la suma de } \\
\text { ambos por separado }\end{array}$ \\
\hline Antagonismo & $\begin{array}{l}\text { Anticoagulantes } \\
\text { orales y vitamina K }\end{array}$ & $\begin{array}{l}\text { Disminuye el efecto } \\
\text { anticoagulante }\end{array}$ \\
\hline
\end{tabular}

administración de la tetraciclina con un vaso de leche como consecuencia de la formación de un complejo insoluble con el calcio que impide la absorción del antibiótico.

También, debido al tratamiento concomitante de un fármaco con un complemento dietético, como es el caso de la Hierba de San Juan con inhibidores selectivos de la recaptación de la serotonina, puede desencadenar sindrome serotoninérgico (4).

\section{Objetivos}

Realizar una revisión general sobre las interacciones farmacológicas centrándonos, en primer lugar, en abordar los diferentes tipos de interacciones implicadas en el fracaso terapéutico.

Revisar el papel del transportador OATP1B1 (transportador de aniones orgánicos polipeptídicos) en el metabolismo de fármacos.

Explicar el mecanismo de interacción entre la Rosuvastatina y el 0lmesartán que deriva en una miositis.

\section{Metodología}

En el presente trabajo se ha llevado a cabo una revisión bibliográfica en la base de datos PubMed en la que se introdujeron las palabras clave “OATP1B1 and interactions" con el objetivo de encontrar artículos centrados en el papel del transportador OATP1B1 en el metabolismo de los xenobióticos y buscar una explicación a la interacción entre la Rosuvastatina (estatina) y el Olmesartán (ARA-II).

Se excluyeron todos aquellos artículos que no estaban relacionados con el transportador objeto de estudio (OATP1B1) y que se centraban exclusivamente en la farmacogenética y en los polimorfismos del OATP1B1 puesto que para este artículo nos interesa el papel que desempeña en el metabolismo de fármacos, las interacciones más relevantes y sus consecuencias a nivel clínico.

También se recopiló información en revistas especializadas en el área de la salud para obtener información sobre la situación actual de los pacientes ancianos polimedicados, en: B0TPlus web, Revistas Elsevier y Geriatricarea. Además, se recurrió a la base de datos Dialnet para encontrar información generalizada sobre las interacciones farmacológicas. 
Tabla 2 Tipos de interacciones farmacocinéticas

\begin{tabular}{|c|c|c|}
\hline $\begin{array}{l}\text { Etapa } \\
\text { farmacocinética }\end{array}$ & $\begin{array}{l}\text { Modificación causada por el fármaco } \\
\text { (alteración) }\end{array}$ & Ejemplo (interacción) \\
\hline \multirow[t]{5}{*}{ Absorción } & Motilidad gastrointestinal & $\begin{array}{l}\text { Metoclopramida y paracetamol (aumento velocidad de absorción } \\
\text { paracetamol) }\end{array}$ \\
\hline & Formación de quelatos & $\begin{array}{l}\text { Ciprofloxacino e hidróxido de aluminio (reducción biodisponibilidad } \\
\text { de ciprofloxacino) }\end{array}$ \\
\hline & Competición por un transportador & Levodopa y fenilalanina (reducción biodisponibilidad levodopa) \\
\hline & $\begin{array}{l}\text { Cambios de pH en el lugar de } \\
\text { absorción }\end{array}$ & $\begin{array}{l}\text { Ketoconazol y omeprazol (disminuye biodisponibilidad ketoconazol } \\
\text { en el } 90 \% \text { ) }\end{array}$ \\
\hline & $\begin{array}{l}\text { Alteración de la membrana de } \\
\text { absorción }\end{array}$ & $\begin{array}{l}\text { Ácido valproico y metotrexato (el metotrexato dificulta la absorción } \\
\text { de ácido valproico) }\end{array}$ \\
\hline Distribución & Unión a proteínas plasmáticas & $\begin{array}{l}\text { Desplazamiento de la digoxina por el ácido salicílico (elevada unión a } \\
\text { proteínas plasmáticas) }\end{array}$ \\
\hline \multirow[t]{2}{*}{ Metabolismo } & Inducción enzimática & $\begin{array}{l}\text { La rifampicina activa el metabolismo enzimático de los anticonceptivos } \\
\text { orales }\end{array}$ \\
\hline & Inhibición enzimática & $\begin{array}{l}\text { El omeprazol actúa (inhibición competitiva) sobre el citocromo } \\
\text { CYP2C19; disminuye la efectividad del clopidogrel (profármaco) }\end{array}$ \\
\hline \multirow[t]{3}{*}{ Excreción } & Modificación del pH urinario & $\begin{array}{l}\text { La acetazolamida alcaliniza la orina y facilita la eliminación del ácido } \\
\text { salicilico. }\end{array}$ \\
\hline & $\begin{array}{l}\text { Alteración de la secreción renal de } \\
\text { fármacos }\end{array}$ & $\begin{array}{l}\text { Probenecid inhibe la secreción renal por transporte activo de los } \\
\text { diuréticos del asa }\end{array}$ \\
\hline & Deterioro de la función renal & $\begin{array}{l}\text { Riesgo de acumulación de fármacos con estrecho margen terapéutico, } \\
\text { como la digoxina }\end{array}$ \\
\hline
\end{tabular}

\section{Resultados y discusión}

Desde que aparece el primer artículo publicado sobre OATP1B1 en el año 2003 hasta nuestros días se han publicado 747 artículos sobre este transportador.

Las proteínas transportadoras de membrana de la familia OATP (Organic Anion Transporting Polypeptide) se han descubierto hace poco más de una década. Constituyen una subfamilia de los transportadores de membrana SLCO (Solute Carrier Organic Anion)

Están implicadas en interacciones farmacológicas de relevancia clínica $\mathrm{y}$ es una de las principales razones por las que varias agencias reguladoras, entre ellas la FDA, recomiendan la evaluación de nuevos fármacos en cuanto al grado de interacción a través de estos transportadores.

Dada la importancia de los OATPs se han revisado estudios recientes para entender mejor el mecanismo de interacción entre la Rosuvastatina y el Olmesartán y, también, para comprender cómo funcionan estos transportadores.

Aparecen publicados en Pubmed 83 artículos con las palabras clave OATP1B1 y rosuvastatine y 6 artícu- los con las palabras clave OATP1B1 y olmesartan y 0 artículos para la combinación con las palabras clave OATP1B1, olmersartan y rosuvastatine.

Las OATP desempeñan un papel importante en la extracción hepática de xenobióticos para su posterior biotransformación y eliminación. Por tanto, se caracterizan por ser unos transportadores secretores y afluentes, que facilitan la captación de sustancias endógenas (sales biliares, hormonas tiroideas...) y de numerosos medicamentos. Las distintas configuraciones que adoptan las proteínas de las OATP originan una serie de isoformas que se clasifican atendiendo a su localización tisular y fármacos que utilizan como sustrato, como se ilustra en la tabla 3 (5).

Actualmente, aún no se ha dilucidado el mecanismo de transporte de los OATPs. Sin embargo, diferentes estudios que se han llevado a cabo en estos últimos años apuntan a que el transporte de sustratos mediado por

Tabla 3 Proteínas que participan en el transporte de ciertos fármacos, y por tanto modifican la absorción, distribución, metabolismo y eliminación de estos

\begin{tabular}{|l|l|l|}
\hline Proteina & Fármacos implicados & Órgano diana \\
\hline OATP1A2 & $\begin{array}{l}\text { Indometacina, rocuronio, enalapril, } \\
\text { rosuvastatina, levofloxacina, metotrexate }\end{array}$ & $\begin{array}{l}\text { Riñón, intestino } \\
\text { delgado y conducto } \\
\text { biliar. }\end{array}$ \\
\hline OATP1B1 & $\begin{array}{l}\text { Rifampicina, atorvastatina, } \\
\text { rosuvastatina, simvastatina, valsartan, } \\
\text { olmesartan, enalapril, caspofungina. }\end{array}$ & $\begin{array}{l}\text { Higado (membrana } \\
\text { basolateral de los } \\
\text { sinusoides). }\end{array}$ \\
\hline OATP1B3 & $\begin{array}{l}\text { Digoxina, rifampicina, enalapril, } \\
\text { fluvastatina, rosuvastatina, valsartan, } \\
\text { metotrexate, paclitaxel. }\end{array}$ & $\begin{array}{l}\text { Higado (membrana } \\
\text { basolateral de los } \\
\text { sinusoides). }\end{array}$ \\
\hline OATP2B1 & $\begin{array}{l}\text { Atorvastatina, pravastatina, pitavastatina, } \\
\text { fluvastatina, rosuvastatina, glibenclamida. }\end{array}$ & Ubicuo. \\
\hline OATP4C1 & Digoxina, metotrexate & Riñón. \\
\hline
\end{tabular}


OATP se rige por un transporte activo secundario en el que interviene el bicarbonato como contraión para favorecer la entrada del sustrato al hepatocito $(6,7)$.

Puesto que se trata de un transporte activo sigue una cinética de Michaelis-Menten. La velocidad de eliminación hepática resulta limitada, y como consecuencia su inhibición por un sustrato competidor, como un fármaco, es un factor crucial y determinante en la aparición de interacciones farmacológicas. De hecho, los estudios que se han revisado apuntan a que actualmente la postura más aceptada es la de una inhibición competitiva del transportador OATP1B1 que afecta a las propiedades farmacocinéticas de un determinado fármaco (7). El estudio dirigido por Alam $\mathrm{K}$ en el presente año señala que varios artículos se han enfocado recientemente en la importancia del transportador 0ATP1B1 en las interacciones farmacológicas con estatinas. Se ha visto que la administración conjunta de las estatinas con fármacos inhibidores del OATP1B1 incrementa los niveles plasmáticos de las estatinas, tal como reflejan los parámetros farmacocinéticos del área bajo la curva (AUC) y la concentración plasmática máxima (Cmax), que se encuentran aumentados cuando se inhibe el transportador. Esto puede generar efectos adversos clínicamente significativos como la miopatía, y la rabdomiolisis, que resulta mortal en casos graves.

Entre los fármacos que inhiben a OATP1B1 se encuentra el Olmesartán, como se observa en la tabla 3 y así lo refiere, también, el artículo de revisión de Alam K.

Estos hechos apoyan la posibilidad de que la tendinitis del paciente debido a una rotura del tendón de Aquiles, y que persistía a pesar de ha- ber realizado varias sesiones de fisioterapia, resultara de una interacción entre el Olmesartán, que actúa como inhibidor del OATP1B1 con la Rosuvastatina (en la tabla 3 se observa también que utiliza el transportador OATP1B1).

\section{Conclusión}

Tras evaluar todos los datos obtenidos y analizar todos los factores que interactúan con el transportador OATP1B1 se concluye que puede producirse una interacción entre el Olmesartán y la Rosuvastatina. Esta interacción se debe a que el Olmesartán parece ser un inhibidor competitivo del transportador OATP1B1, además de usarlo para su metabolismo, lo que afecta al metabolismo de la Rosuvastatina. Esto provoca un aumento de la concentración de la Rosuvastatina en sangre, con lo cual, debería reducirse la dosis de administración de Rosuvastatina. De esta manera, evitaríamos posibles efectos adversos derivados de la interacción.

\section{Referencias bibliográficas}

1. Anónimo. La importancia real de las interacciones. Panorama Actual Med 2001; 25(245): 583-590 [acceso noviembre 2018]. Disponible en: http://publicaciones.portalfarma.com/ pam/245/revision_1.pdf

2. Garrido-Garrido EM, García-Garrido I, García-López-Durán JC, García-Jiménez F, Ortega-López I, Bueno-Cavanillas A. Estudio de pacientes polimedicados mayores de 65 años en un centro de asistencia primaria urbano. Rev Calid Asist. 2011; 26(2): 90-96. [acceso noviembre 2018]. Disponible en http:// www.elsevier.es/es-revista-revista-calidad-asistencial-256-articulo-estudio-pacientes-polimedicados-mayores-65-S1134282X10001454

3. Teresa Molina López, María de la O Caraballo Camacho, Soledad López Rubio
Juan CarlosDomínguez Camacho, Juan Carlos Morales Serna. Prevalencia de polimedicación y riesgo vascular en la población mayor de 65 años. Atención Primaria 2012; 44 (4):216-222 [acceso noviembre 2018]. Disponible en https://www.sciencedirect.com/science/ article/pii/S021265671100360X

4. Barcia-Hernández E, Negro-Alvarez S. Fundamentos de las interacciones farmacocinéticas. Anal Real Acad Farm. 2002; 68: 126-172. [acceso noviembre 2018]. Disponible en https://www.analesranf.com/index.php/aranf/article/ viewFile/158/191

5. Ojeda GD. Papel de los transportadores en la Farmacología. 2015. [acceso noviembre 2018]. Disponible en: https://www.researchgate.net/ publication/279181463_Papel_de_ los_transportadores_en_la_Farmacologia

6. Russel FGM. Transporters: importance in drug absorption, distribution, and removal. En: K.S. Pang et al. (eds.), Enzyme and TransporterBased Drug-Drug Interactions. American Association of Pharmaceutical Scientists. 2010. DOI 10.1007/978-1-4419-0840-7_2 [асceso noviembre 2018]. Disponible en: https://www.google.com/url $\mathrm{sa}=\mathrm{tCt} \mathrm{rct}=\mathrm{jEtq}=\mathrm{Etesrc}=\mathrm{st}$ souce $=$ web $\mathrm{tcd}=1 \mathrm{Ctved}=2$ ahUKEwNop Tny 4TfAhWjAhAIHS 4ln 8QFj AAegQIAxACCturl=htps $\% 3 \mathrm{~A} \% 2$ $\mathrm{F} \% 2 \mathrm{Fwww}$.springercom $\% 2 \mathrm{Fcd}$ a $\% 2$ Fcontent $\% 2 \mathrm{Fdcument} \% 2 \mathrm{~F}$ cda_downloaddcument\%2F97 81441908391-c1pdf\%3FSGWID \%3D0-0-45-837961-p 1739067 08ctusg $=\mathrm{A0vVaw0DnFOuPqNAU}$ VWexMw_65wl

7. Alam K, Crowe A, Wang X, Zhang P, Ding K, Li L. Regulation of organic anion transporting polypeptides (OATP)1B1 and OATP1B3- mediated transport: an updated review in the context of OATP-mediated drug-drug interactions. 2018; Int J Mol Sci.. 19 (3), 855 [acceso noviembre 2018] Disponible en https://www.mdpi.com/1422 $-0067 / 19 / 3 / 855$ 